\title{
Deflection of Beams by Means of Static Green Functions
}

\author{
M. Abu-Hilal \\ Department of Mechanical Engineering, Zarqa University, Jordan
}

Copyright $(2016$ by authors, all rights reserved. Authors agree that this article remains permanently open access under the terms of the Creative Commons Attribution License 4.0 International License

\begin{abstract}
A Green's Function method for determining the deflection of statically loaded beams is presented. This method may be applied to single and multi-span, statically determinate and indeterminate beams subjected to single or several loads. Also Green's functions for different beams are given. Finally, several examples are given to illustrate the use of Green's functions.
\end{abstract}

Keywords Green Function, Deflection of Beams, Static Load

\section{Introduction}

The deflection $v(x)$ of a beam can be determined by the integration method or the use of discontinuity functions, [1] and [2]. To find the deflection of a beam by the method of integration, we integrate the moment equation $E I v^{\prime \prime}=$ $M(x)$ twice, which requires the knowledge of $M(x)$ as a continuous function throughout the beam's entire length and the introducing of integration constants. These integration constants are then evaluated using the boundary conditions of the considered beam. The method of integration is convenient for single-span beams with single load. If the loading on the beam consists of several distributed and concentrated loads acting on different positions on the beam, the method of integration becomes more tedious to apply, since separate moment functions have to be given for each region between the loads [1]. Also integration of these moment functions requires the evaluation of two integration constants for each region using boundary and/or continuity conditions.

The method of discontinuity functions is particularly advantageous for solving problems involving multiply loaded beams. By this method, discontinuity functions are used to give the internal moment $\mathrm{M}(\mathrm{x})$ as a single expression, independent of the number of loads acting on the beam. Integrating the moment equation $E I v^{\prime \prime}=M(x)$ twice yields an expression for the deflection of the beam with only two integration constants included. These constants can then be evaluated by using only the boundary conditions. Note that by the use of the aforementioned methods we have to find the support reactions and the internal moments before we can find the deflection of the beam.

In this paper a method using Green's functions for determining the deflection of statically loaded prismatic beams is presented. This method may be used for single and multi-span beams, single and multi loaded beams, and statically determinate and indeterminate beams. The method of Green functions is easier to use than the aforementioned methods because this method does not require determining the support reactions or the internal moment expressions of the considered beam. Furthermore, since no integration constants occur in the integrated Green's functions, we don't need to consider the boundary or the continuity conditions for the statically determinate beams. The method of Green's functions is particularly convenient for solving problems involving multi-loaded or statically indeterminate beams. Also, Green functions for different beams are determined. In order to demonstrate the use of the Green functions method, several application examples are given.

\section{Green's Functions for Statically Loaded Beams}

A Green's function of a beam is its response at a point $x$ due to unit concentrated force acting on an arbitrary position $\xi$ between the two ends of the beam. Thus Green's functions and impulse responses are identical, except that the former are involved in the solutions to boundary value problems, whereas the latter are involved in solutions to initial value problems [3]. In the following, Green's functions for elastic homogeneous isotropic uniform beams with different boundary conditions will be derived using Laplace transform.

The elastic deflection $v(x)$ of an initially straight prismatic beam due to a static load can be described by the differential equation

$$
E I v^{\prime \prime \prime \prime}(x)=-w(x),
$$

where $E I$ is the bending rigidity of the beam. The deflection $v(x)$ is positive upward and the $x$-axis extends positive to the right, along the initially straight longitudinal axis of the beam as shown in Fig. 1. A prime denotes derivative with respect to $x$. 


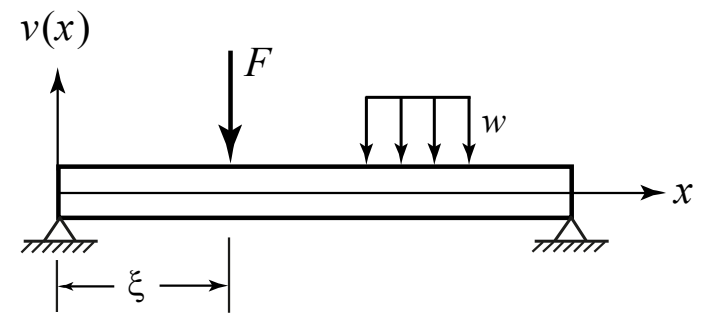

Figure1. Deflection of beams

The applied distributed load $w(x)$ is positive in the downward direction. By the action of a concentrated force $F$ at a position $\xi$, the load $w$ is given as

$$
w(x)=F \delta(x-\xi)
$$

and by the action of a moment load $M_{0}$ at a position $\xi$, the load $w$ is given as

$$
w(x)=M_{0} \delta^{\prime}(x-\xi),
$$

where $\delta(\cdot)$ is the Dirac delta function.

In order to determine the Green's function for a beam, we replace the acting arbitrary distributed load $w(x)$ with a unit concentrated force $(F=1)$ applied at an arbitrary position $\xi$ between the two ends of the beam as shown in Fig. 1. Hence Eq. (1) becomes

$$
\operatorname{EIV}^{\prime \prime \prime \prime}(x)=-\delta(x-\xi) .
$$

Laplace transforming this equation yields

$$
\begin{aligned}
& E I\left[s^{4} V(s)-s^{3} v(0)-s^{2} v^{\prime}(0)-\right. \\
& \left.-s v^{\prime \prime}(0)-v^{\prime \prime \prime}(0)\right]=-e^{-s \xi}
\end{aligned}
$$

From this equation, the Laplace transform of the deflection is obtained as

$$
V(s)=\frac{v^{\prime \prime \prime}(0)}{s^{4}}+\frac{v^{\prime \prime}(0)}{s^{3}}+\frac{v^{\prime}(0)}{s^{2}}+\frac{v(0)}{s}-\frac{e^{-s \xi}}{E I s^{4}} .
$$

Inverting this equation leads to

$$
\begin{aligned}
& g(x, \xi)=\frac{v^{\prime \prime \prime}(0)}{6} x^{3}+\frac{v^{\prime \prime}(0)}{2} x^{2}+v^{\prime}(0) x+ \\
& +v(0)-\frac{(x-\xi)^{3}}{6 E I} u(x-\xi)
\end{aligned}
$$

where $u(x-\xi)$ is the unit step function defined as

$$
u(x-\xi)=\left\{\begin{array}{ll}
0 & x<\xi \\
1 & x>\xi
\end{array} .\right.
$$

Equation (7) is called the Green's function of Eq. (1) and represents the deflection of point $x$ on the beam when a unit load has been applied at an arbitrary point $\xi$ between the two ends of the beam.

Once the Green's function for the beam is obtained, the deflection of the beam due to any arbitrary loading distribution may be given in the form

$$
v(x)=\int_{0}^{L} w(\xi) g(x, \xi) d \xi,
$$

where $L$ is the length of the beam and $w(\xi) g(x, \xi) d \xi$ is the incremental deflection at $x$ due to the actual load intensity at $\xi$,[3]. Depending upon the number of the acting loads on the beam and the number of the beam's spans, the integral in Eq. (9) has to be divided into several integrals (see the following examples).

The unknown parameters $\mathrm{v}(0), v^{\prime}(0), v^{\prime \prime}(0)$, and $v^{\prime \prime \prime}(0)$ in Eq. (7) are to be determined from the boundary conditions. As an example, for a simply supported beam, the deflection and internal moment must vanish at $x=0$ and $x=L$. Thus, the boundary condition at $x=0$ are

$$
v(0)=0, \quad v^{\prime \prime}(0)=0,
$$

and at $x=L$ are

$$
v(L)=0, \quad v^{\prime \prime}(L)=0 .
$$

Substituting Eq. (10) into Eq. (7) yields

$$
v(x, \xi)=\frac{v^{\prime \prime \prime}(0)}{6} x^{3}+v^{\prime}(0) x-\frac{(x-\xi)^{3}}{6 E I} u(x-\xi) .
$$

Using Eq. (11) yields the following two equations

$$
\begin{gathered}
v(L, \xi)=\frac{v^{\prime \prime \prime}(0)}{6} L^{3}+v^{\prime}(0) L-\frac{(L-\xi)^{3}}{6 E I}=0, \\
v^{\prime \prime}(L, \xi)=v^{\prime \prime \prime}(0) L-\frac{(L-\xi)}{E I}=0 .
\end{gathered}
$$

Solving these equations yields for the parameters $v^{\prime}(0)$ and $v^{\prime \prime \prime}(0)$ :

$$
\begin{gathered}
v^{\prime}(0)=\frac{-1}{6 E I L}\left[\xi^{3}-3 L \xi^{2}+2 L^{2} \xi\right], \\
v^{\prime \prime \prime}(0)=\frac{L-\xi}{E I L} .
\end{gathered}
$$

Substituting Eq. (15) and Eq. (16) into Eq. (12) yields the Green's function for a simply supported beam in the form

$$
\begin{aligned}
& g(x, \xi)=\frac{1}{6 E I L}\left[(L-\xi) x^{3}-\left(\xi^{3}-3 L \xi^{2}+2 L^{2} \xi\right) x\right]- \\
& -\frac{(x-\xi)^{3}}{6 E I} u(x-\xi)
\end{aligned}
$$

or in intervals form

$$
g(x, \xi)=\left\{\begin{array}{ll}
\frac{1}{6 E I L}\left[(L-\xi) x^{3}-\left(\xi^{3}-3 L \xi^{2}+2 L^{2} \xi\right) x\right] & x \leq \xi \leq L . \\
\frac{1}{6 E I L}\left[(L-\xi) x^{3}-\left(\xi^{3}-3 L \xi^{2}+2 L^{2} \xi\right) x\right]-\frac{(x-\xi)^{3}}{6 E I} & 0 \leq \xi \leq x
\end{array} .\right.
$$

Table 1 in the Appendix includes Green's functions for beams with different boundary conditions derived by using Eq. (7) and appropriate boundary conditions. 


\section{Application Examples}

The following examples demonstrate the use of Green's functions for determining the deflection of different beams

\subsection{Simply Supported Beam with Concentrated Force}

Figure 2 shows a simply supported beam subjected to a concentrated force, $w(x)=F \delta(x-a)$. The deflection of this beam may be obtained directly from Eq. (18) as

$$
v(x)=\left\{\begin{array}{ll}
v_{1}=\frac{F}{6 E I L}\left[(L-a) x^{3}-a\left(a^{2}-3 a L+2 L^{2}\right) x\right] & 0 \leq x \leq a \\
v_{2}=v_{1}-\frac{(x-a)^{3}}{6 E I} & a \leq x \leq L
\end{array},\right.
$$

or in a simpler form

$$
v(x)=\left\{\begin{array}{ll}
\frac{F b x}{6 E I L}\left(x^{2}+b^{2}-L^{2}\right) & 0 \leq x \leq a \\
\frac{F a(L-x)}{6 E I L}\left(x^{2}+a^{2}-2 L x\right) & a \leq x \leq L
\end{array} .\right.
$$

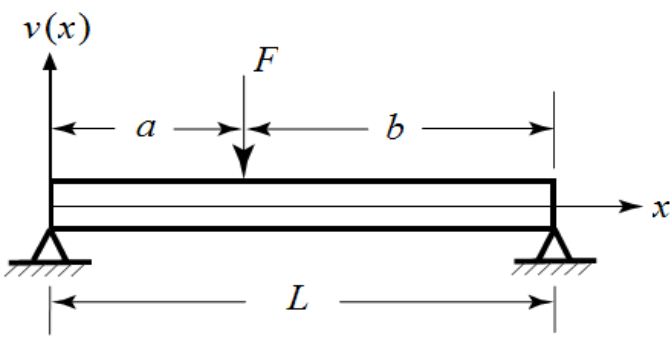

Figure 2. Simply supported beam with concentrated force

\subsection{Simply Supported Beam with Distributed Load}

Using the unit step function, the acting load on the beam shown in Fig. 3 may be given as

$$
w(x)=w_{0}[u(x-a)-u(x-b)] .
$$

Substituting this equation and Eq. (17) into Eq. (9) gives

$$
\begin{aligned}
v(x)= & \frac{w_{0}}{6 E I L} \int_{0}^{L}\left[(L-\xi) x^{3}-\left(\xi^{3}-3 L \xi^{2}+2 L^{2} \xi\right) x\right][u(\xi-a)-u(\xi-b)] d \xi \\
& -\frac{w_{0}}{6 E I} \int_{0}^{L}(x-\xi)^{3} u(x-\xi)[u(\xi-a)-u(\xi-b)] d \xi \cdot(22)
\end{aligned}
$$

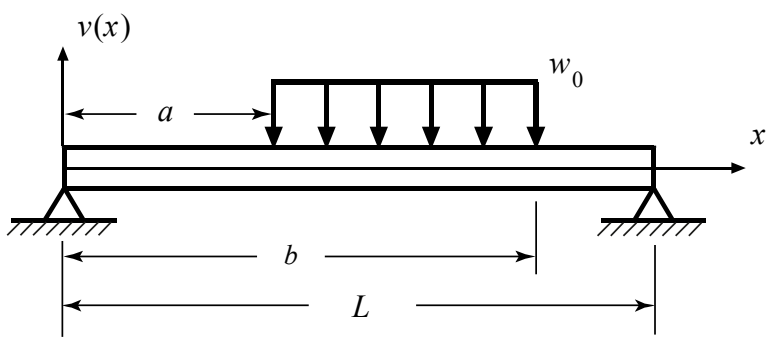

Figure 3. Simply supported beam with constant distributed load

The first integral in this equation is to be integrated only between $a$ and $b$, since the load is equal to zero for $x<a$ and $x>b$, thus

$$
\begin{gathered}
v(x)=\frac{w_{0}}{6 E I L}\left\{\left[(b-a) L-\frac{1}{2}\left(b^{2}-a^{2}\right)\right] x^{3}-\right. \\
\left.\left[\frac{1}{4}\left(b^{4}-a^{4}\right)-\left(b^{3}-a^{3}\right) L+\left(b^{2}-a^{2}\right) L^{2}\right] x\right\}- \\
-\frac{w_{0}}{6 E I} \int_{0}^{L}(x-\xi)^{3} u(x-\xi)[u(\xi-a)-u(\xi-b)] d \xi
\end{gathered}
$$
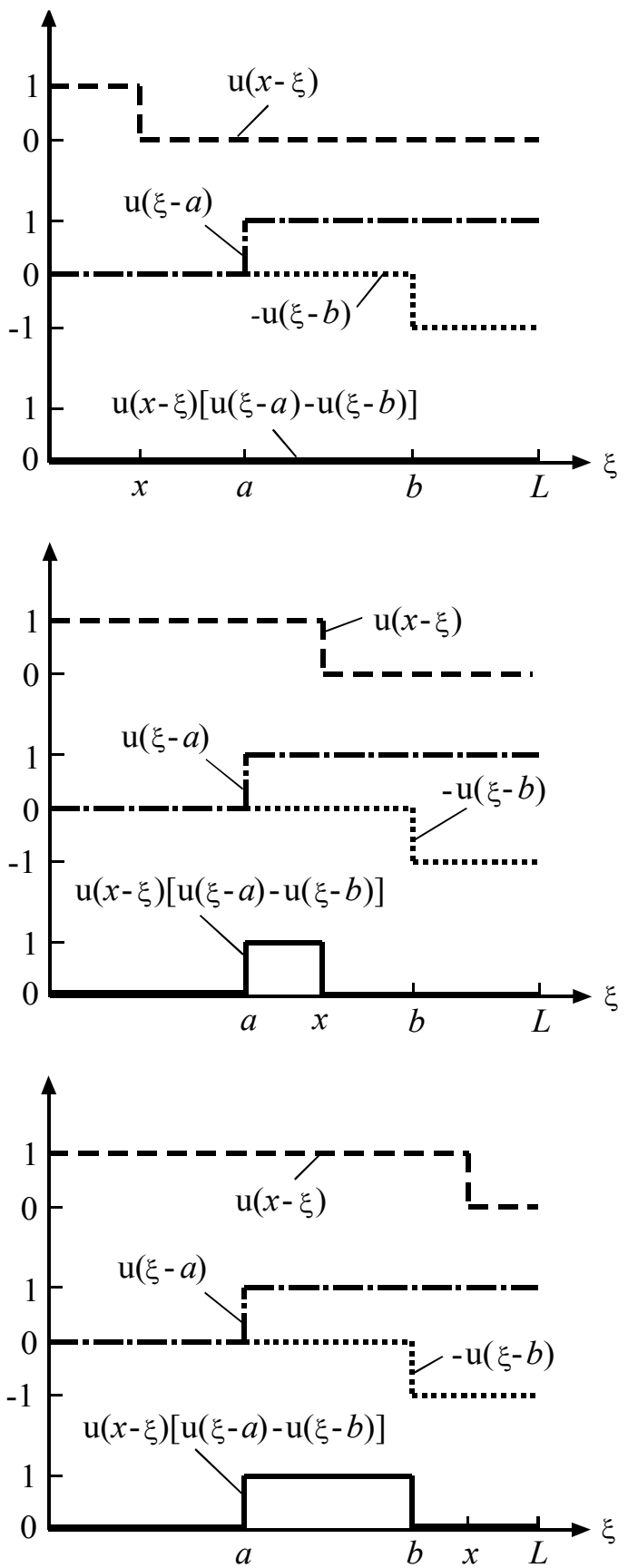

Figure 4. Product of step functions for example 3

Because of the three step functions in the remaining integral in this equation, this integral is to be divided into three separate integrals, depending upon whether $0 \leq x \leq a, a$ $\leq x \leq b$, or $b \leq x \leq L$. For this reason we use Fig. 4 which gives for the product of the step functions $u(x-\xi)[u(\xi-a)-u(\xi-b)]$ for 
all $\xi$ over the interval $0 \leq \xi \leq L$ :

- the value 0 as long as $x<a$,

- the value 0 except for $a \leq \xi \leq x$ where the product is 1 , and

- the value 0 except for $a \leq \xi \leq b$ where the product is 1 .

Thus we obtain for the second integral in Eq. (22) the value of 0 for $0 \leq x \leq a$.

For the interval $a \leq x \leq b$, we get

$$
\int_{a}^{x}(x-\xi)^{3} d \xi=\frac{x^{4}}{4}-a x^{3}+\frac{3}{2} a^{2} x^{2}-a^{3} x+\frac{a^{4}}{4} .
$$

Finally, for the interval $b \leq x \leq L$, we obtain

$$
\int_{a}^{b}(x-\xi)^{3} d \xi=(b-a) x^{3}-\frac{3}{2}\left(b^{2}-a^{2}\right) x^{2}+\left(b^{3}-a^{3}\right) x-\frac{1}{4}\left(b^{4}-a^{4}\right) \text {. }
$$

Combining the results in Eqs. (23), (24), and (25) yields the final solution in the form:

$$
\begin{gathered}
v(x)=\frac{w_{0}}{6 E I L}\left\{\left[(b-a) L-\frac{1}{2}\left(b^{2}-a^{2}\right)\right] x^{3}-\left[\frac{1}{4}\left(b^{4}-a^{4}\right)-\left(b^{3}-a^{3}\right) L+\left(b^{2}-a^{2}\right) L^{2}\right] x\right\} \\
-\frac{w_{0}}{6 E I}\left\{\frac{x^{4}}{4}-a x^{3}+\frac{3}{2} a^{2} x^{2}-a^{3} x+\frac{a^{4}}{4}\right\} u(x-a) \\
-\frac{w_{0}}{6 E I}\left\{(b-a) x^{3}-\frac{3}{2}\left(b^{2}-a^{2}\right) x^{2}+\left(b^{3}-a^{3}\right) x-\frac{1}{4}\left(b^{4}-a^{4}\right)\right\} u(x-b),
\end{gathered}
$$

or in the form

$$
v(x)=\frac{w_{0}}{24 E I L} \begin{cases}c_{1} x^{3}+c_{2} x & 0 \leq x \leq a \\ -L x^{4}+c_{3} x^{3}-6 L a^{2} x^{2}+c_{4} x-L a^{4} & a \leq x \leq b \\ -2\left(b^{2}-a^{2}\right) x^{3}+6 L\left(b^{2}-a^{2}\right) x^{2}-c_{5} x+c_{6} & b \leq x \leq L\end{cases}
$$

where

$$
\begin{gathered}
c_{1}=4 L(b-a)-2\left(b^{2}-a^{2}\right), \\
c_{2}=4 L\left(b^{3}-a^{3}\right)-\left(b^{4}-a^{4}\right)-4 L^{2}\left(b^{2}-a^{2}\right), \\
c_{3}=4 L b-2\left(b^{2}-a^{2}\right) \\
c_{4}=4 L b^{3}-b^{4}+a^{4}-4 L^{2}\left(b^{2}-a^{2}\right) \\
c_{5}=b^{4}-a^{4}-4 L^{2}\left(b^{2}-a^{2}\right) \\
c_{6}=L\left(b^{4}-a^{4}\right) .
\end{gathered}
$$

\subsection{Simply Supported Beam with Moment Load $M_{0}$}

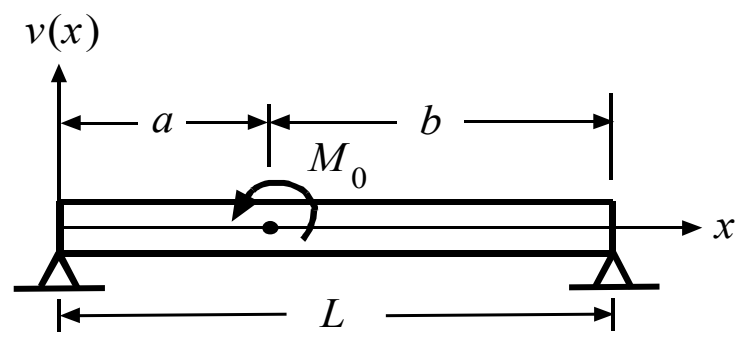

Figure 5. Simply supported beam with moment load
In this example we consider a simply supported beam subjected to concentrated moment load as shown in Fig. 5.

The load may be described corresponding to Eq. (3) as

$$
w(x)=M_{0} \delta^{\prime}(x-a) .
$$

Substituting this equation and Eq. (17) into Eq. (9) gives

$$
\begin{aligned}
v(x) & =\frac{M_{0}}{6 E I L} \int_{0}^{L}\left[(L-\xi) x^{3}-\left(\xi^{3}-3 L \xi^{2}+2 L^{2} \xi\right) x\right] \delta^{\prime}(\xi-a) d \xi \\
& -\frac{M_{0}}{6 E I} \int_{0}^{L}(x-\xi)^{3} u(x-\xi) \delta^{\prime}(\xi-a) d \xi .
\end{aligned}
$$

Using formal integral, Making use of the relation

$$
\int_{a}^{c} \delta^{(n)}(\xi-b) f(\xi) d \xi=(-1)^{n} f^{(n)}(b) \quad a \leq b \leq c,
$$

gives for the deflection in Eq. (28):

$$
\begin{aligned}
& v(x)=\frac{M_{0} x}{6 E I L}\left(x^{2}+3 a^{2}+2 L^{2}-6 a L\right) x- \\
& -\frac{M_{0}}{2 E I}(x-a)^{2} u(x-a)
\end{aligned},
$$

or in a more familiar form

$$
v(x)=\frac{M_{0}}{6 E I L} \begin{cases}\left(x^{2}+3 a^{2}+2 L^{2}-6 a L\right) x & 0 \leq x \leq a \\ x^{3}-3 L x^{2}+\left(3 a^{2}+2 L^{2}\right) x-3 a^{2} L & a \leq x \leq L\end{cases}
$$

\subsection{Statically Indeterminate, Two-span Beam}

We consider in this example the statically indeterminate beam shown in Fig. 6. The support at $B$ as an external force which can be determined after the deflection has been found from the condition $v(b)=0$. Thus, the acting load may be given as

$$
w(x)=F_{1} \delta(x-a)-B \delta(x-b)+F_{2} \delta(x-L) .
$$

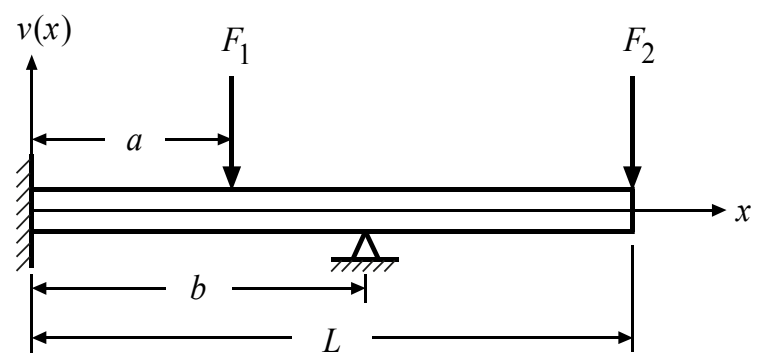

Figure 6. Statically indeterminate cantilevered beam

The Green's function for a cantilevered beam is obtained from Table 1 as

$$
g(x, \xi)=\frac{x^{2}}{6 E I}(x-3 \xi)-\frac{(x-\xi)^{3}}{6 E I} u(x-\xi) .
$$

Substituting Eqs. (32) and (33) into Eq. (9) yields 
$v(x)=\int_{0}^{L} \frac{x^{2}}{6 E I}(x-3 \xi)\left[F_{1} \delta(\xi-a)-B \delta(\xi-b)+F_{2} \delta(\xi-L)\right] d \xi$ $-\int_{0}^{L} \frac{(x-\xi)^{3}}{6 E I}\left[F_{1} \delta(\xi-a)-B \delta(\xi-b)+F_{2} \delta(\xi-L)\right] u(x-\xi) d \xi$.

The first integral in this equation can be readily evaluated by using the relation

$$
\int_{a}^{c} f(\xi) \delta(\xi-b) d \xi=\left\{\begin{array}{ccc}
0 & \text { for } & b<a<c \\
f(b) & \text { for } & a<b<c \\
0 & \text { for } & a<c<b
\end{array}\right.
$$

Thus, we obtain for Eq. (34)

$$
\begin{gathered}
v(x)=\frac{x^{2}}{6 E I}\left[F_{1}(x-3 a)-B(x-3 b)+F_{2}(x-3 L)\right] \\
-\int_{0}^{L} \frac{(x-\xi)^{3}}{6 E I}\left[F_{1} \delta(x-a)-B \delta(x-b)+F_{2} \delta(x-L)\right] u(x-\xi) d \xi .
\end{gathered}
$$

The evaluation of the integral in this equation gives three solutions depending upon the product of the step functions included in the integral. This product gives for all $\xi$ over the interval $0 \leq \xi \leq L$ the value of 0 for $0 \leq x \leq a, F_{1} \delta(\xi-a)$ for $a \leq$ $x \leq b$, and $F_{1} \delta(\xi-a)-B \delta(\xi-b)$ for $b \leq x \leq L$. Thus, using Eq. (35), the integral in Eq. (36) gives the value 0 for $0 \leq x \leq a$, $\frac{-F_{1}}{6 E I}(x-a)^{3}$ for $a \leq x \leq b$, and $\frac{-1}{6 E I}\left[F_{1}(x-a)^{3}-B(x-b)^{3}\right]$ for $b \leq x \leq L$. Combining all the obtained results gives for the deflection of the beam shown in Fig. 6:

$v(x)=\frac{1}{6 E I}\left\{\begin{array}{ll}{\left[F_{1}(x-3 a)-B(x-3 b)+F_{2}(x-3 L)\right] x^{2}} & 0 \leq x \leq a \\ F_{1} a^{2}(a-3 x)-B(x-3 b) x^{2}+F_{2}(x-3 L) x^{2} & a \leq x \leq b \\ F_{1} a^{2}(a-3 x)-B b^{2}(b-3 x)+F_{2}(x-3 L) x^{2} & b \leq x \leq L\end{array}\right.$.

The unknown support reaction $B$ can be obtained from the condition $v(b)=0$. This condition gives for $B$ the value:

$$
B=\frac{(3 b-a) a^{2} F_{1}+(3 L-b) b^{2} F_{2}}{2 b^{3}} .
$$

\section{Acknowledgements}

This Research is funded by the Deanship of Scientific

\begin{tabular}{|c|c|}
\hline Beam Type & $g(x, \xi)$ \\
\hline$\Delta$ & $\frac{1}{6 E I L}\left[(L-\xi) x^{3}+\left(3 L \xi^{2}-\xi^{3}-2 L^{2} \xi\right) x\right]-\frac{(x-\xi)^{3}}{6 E I} u(x-\xi)$ \\
\hline & $\frac{x^{2}}{6 E I L^{3}}\left[\left(L^{3}-3 L \xi^{2}+2 \xi^{3}\right) x-3 L \xi\left(\xi^{2}-2 L \xi+L^{2}\right)\right]-\frac{(x-\xi)^{3}}{6 E I} u(x-\xi)$ \\
\hline$\triangle$ & $\frac{x^{2}}{12 E I L^{3}}\left[\left(2 L^{3}-3 L \xi^{2}+\xi^{3}\right) x-3 L \xi\left(\xi^{2}-3 L \xi+2 L^{2}\right)\right]-\frac{(x-\xi)^{3}}{6 E I} u(x-\xi)$ \\
\hline$\Delta$ & $\frac{x}{12 E I L^{3}}\left[\left(2 L^{3}-3 L \xi^{2}+\xi^{3}\right) x^{2}-3 L^{2} \xi\left(L^{2}-2 L \xi+\xi^{2}\right)\right]-\frac{(x-\xi)^{3}}{6 E I} u(x-\xi)$ \\
\hline & $\frac{1}{6 E I}\left[x^{3}-3 x^{2} \xi\right]-\frac{(x-\xi)^{3}}{6 E I} u(x-\xi)$ \\
\hline & $\frac{1}{6 E I}\left[3(L-\xi)^{2} x-2 L^{3}+3 L^{2} \xi-\xi^{3}\right]-\frac{(x-\xi)^{3}}{6 E I} u(x-\xi)$ \\
\hline$\Lambda$ & $\frac{x}{6 E I}\left[x^{2}+3 \xi(\xi-2 L)\right]-\frac{(x-\xi)^{3}}{6 E I} u(x-\xi)$ \\
\hline $\mid$ & $\frac{x^{2}}{12 E I L}[2 L x+3 \xi(\xi-2 L)]-\frac{(x-\xi)^{3}}{6 E I} u(x-\xi)$ \\
\hline
\end{tabular}
Research in Zarqa University/Jordan.

\section{Appendix}

Table 1 A. Static Green functions for different beams 


\section{REFERENCES}

[3] L. A. Pipes and L. R. Harvill. Applied mathematics for Engineers and Physicists, $3^{\text {rd }}$ Edition, McGraw-Hill, Tokyo, 1971.

[1] R. C. Hibbeler. Mechanics of materials, $9^{\text {th }}$ edition, Prentice-Hall, U.S.A.,2013.

[2] A. Higdon et al., Mechanics of Materials, $4^{\text {th }}$ edition, John

[4] M. Abu-Hilal, Forced vibration of Euler-Bernoulli beams by means of dynamic Green functions, Elsevier Science Ltd., Journal of Sound and Vibration, Vol. 267, No 2, 191-207, 2003. 